American Journal of Applied Sciences 5 (6): 721-725, 2008

ISSN 1546-9239

(C) 2008 Science Publications

\title{
Microstructural Study of Al-Si-Mg Alloy Reinforced with Stainless Steel Wires Composite via Casting Technique
}

\author{
Mazlee Mohd. Noor, Shamsul Baharin Jamaludin and Kamarudin Hussin \\ School of Materials Engineering, University Malaysia Perlis (UniMAP), \\ P.O. Box 17, Pejabat Pos Kangar, 01007 Kangar, Perlis, Malaysia
}

\begin{abstract}
The microstructural study of Al-Si-Mg alloy reinforced with stainless steel wires $\left(\mathrm{Al}-\mathrm{Si}-\mathrm{Mg} / \mathrm{SS}_{\mathrm{w}}\right.$ ) has been investigated and focused on the both microstructures in the matrix and near to the interface and also the wetting condition at the interface. The wettability of solid metals by liquid metals is very important in determining the needed properties of the composite systems. In this study, the wetting conditions have been characterized using optical microscopy and scanning electron microscopy. Good wetting, incomplete wetting and very poor wetting associated with large voids are seen at the $\mathrm{Al}-\mathrm{Si}-\mathrm{Mg} / \mathrm{SS}_{\mathrm{w}}$ interfaces. Heavily concentrated of needle shaped eutectic Si particles are found near to the single stainless steel wire as compared to double stainless steel wires.
\end{abstract}

Key words: Interface, wetting conditions, needle shaped eutectic Si particles

\section{INTRODUCTION}

The unique properties of the fiber reinforced composite materials are to a great extent dependent on the unique nature on the matrix-fiber interface which can be defined as the region of significantly changed chemical composition constituting the bond between the matrix and the reinforcement for transfer of loads between these members of the composite structure ${ }^{[1]}$. Interfaces constitute an important microstructural feature of composite materials. Fabrication technique and optimum fabrication parameters play the crucial roles in determining the resulted fiber-matrix interface. Major fabrication methods used for aluminum metal matrix composites are stir casting ${ }^{[2,3]}$, squeeze casting $^{[4,5]}$, rheocasting ${ }^{[6,7]}$, infiltration ${ }^{[8,9]}$ and powder metallurgy ${ }^{[10-12]}$.

Recently, aluminum-stainless steel composites have gained the attention due to the low cost of fabrication, the sustainability issue of the stainless steel and the potential to be used particularly for wear applications. Only few researches regarding microstructure have been conducted on the aluminumstainless steel composites system via die casting technique $^{[13]}$, liquid infiltration technique ${ }^{[14]}$ and vortex technique $^{[15]}$.

The wettability of solid metals by liquid metals is very important in determining the needed properties of the material systems. Wettability can be defined as the ability of the liquid to spread on a solid surface. It also describes the extent of intimate contact between a liquid and a solid ${ }^{[16]}$. No attempt so far was made to study on microstructure of wetting conditions of Al-Si-Mg alloy reinforced with stainless steel wire composite. This research presents the study on both microstructures in the matrix and near to the interface and wetting conditions of $\mathrm{Al}-\mathrm{Si}-\mathrm{Mg}$ alloy reinforced with stainless steel wires composite via casting technique.

\section{MATERIALS AND METHOD}

The matrix alloy used in this present work was primary cast ingot Al-Si-Mg alloy (Al-7.3 wt\% Si- $0.24 \mathrm{wt} \% \mathrm{Mg}$ ) and stainless steel wires of $500 \mu \mathrm{m}$ diameter were used as the reinforcement. Stainless steel wires $\left(\mathrm{SS}_{\mathrm{w}}\right)$ were aligned at the uniform distance and fixed on a stainless steel mould of $30 \mathrm{~mm}$ height and $90 \mathrm{~mm}$ diameter.

The matrix alloy was melted in a furnace using clay crucible. Matrix alloy was heated up to the superheating temperature of $850^{\circ} \mathrm{C}$ before poured into the preheated stainless steel mould. The microstructure in the matrix was observed by light microscopy. The microstructures in the matrix and near to the interface were studied by Scanning Electron Microscopy (SEM) at high magnification under mode of secondary electron. The present chemical elements in the microstructural features were determined using energy dispersive X-ray spectroscopy (EDS).

Corresponding Author: Mazlee Mohd. Noor, School of Materials Engineering, University Malaysia Perlis (UniMAP), P.O. Box 17, Pejabat Pos Kangar, 01007 Kangar, Perlis, Malaysia 


\section{RESULTS AND DISCUSSION}

Figure 1 indicates the solidification of $\mathrm{Al}-\mathrm{Si}-\mathrm{Mg}$ matrix alloy into the forms of structure which re globular structures and needle shaped eutectic $\mathrm{Si}$ structures. This optical micrograph of single stainless steel wire shows the majority of the structure is globular structures (denoted by G) meanwhile the needle shaped eutectic Si structures (denoted by N) are segregated at the grain boundaries in the $\mathrm{Al}-\mathrm{Si}-\mathrm{Mg}$ matrix alloy and the incomplete wetting is seen at the interface.

In Fig. 2, the optical micrograph of double stainless steel wires shows incomplete wetting at the interface and good wetting are observed and labeled by I and C, respectively. The porosities (irregular black areas) also found in the Al-Si-Mg matrix alloy which is far away from the double stainless steel wires.

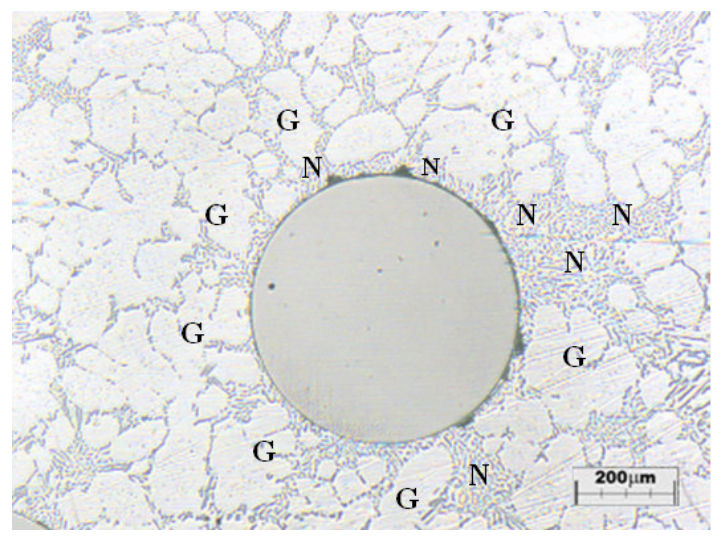

Fig. 1: Optical micrograph of single stainless steel wire

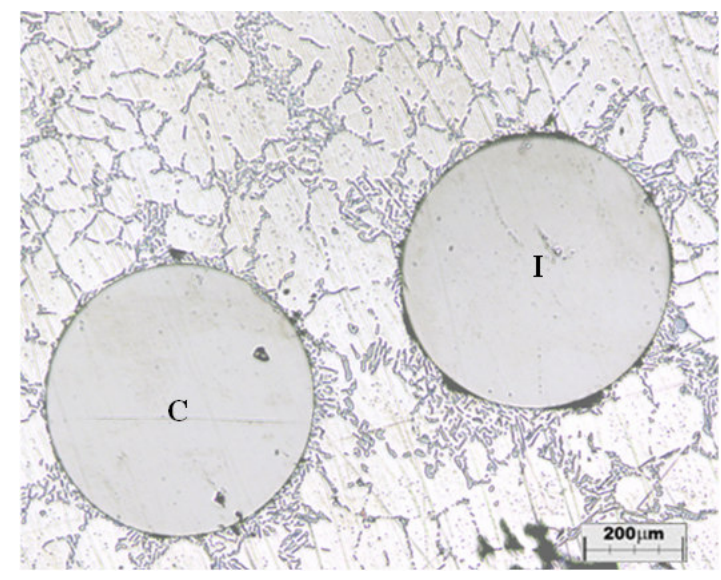

Fig. 2: Optical micrograph of double stainless steel wires
Heavily concentrated of needle shaped eutectic $\mathrm{Si}$ particles near to the single stainless steel wire was observed in Fig. 1 compared to the double stainless steel wires in Fig. 2. In addition, globular structure can be seen in a larger size around single stainless steel wire compared to that of double stainless steel wires. These features are may be attributing to the faster solidification rate around the double stainless steel wires compared to the single stainless steel wire.

Figure 3a shows the micrograph of the good wetting at single stainless steel wire. The stainless steel wire surrounds with the minority of the eutectic $\mathrm{Si}$ needle shaped particles near to the interface and the majority of the globular structure at far from the interface.

The morphology which closes to the interface can be seen as eutectic Si needle shaped particle in Fig. 3b. However, far away from the interface, this morphology

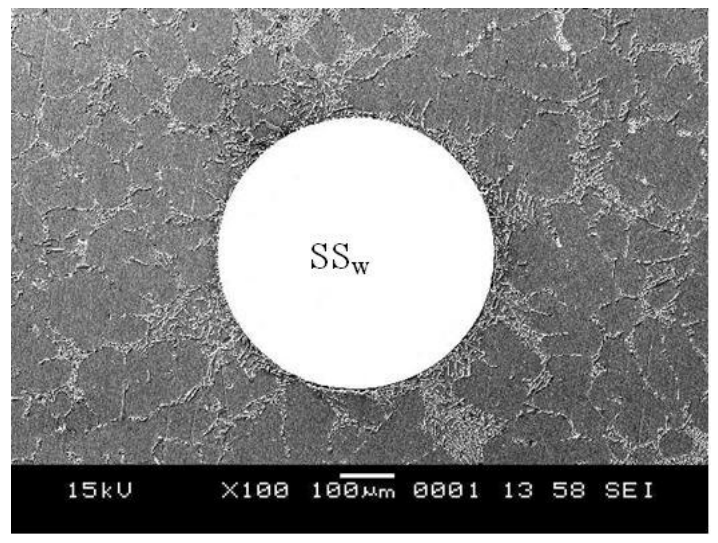

Fig. 3a: SEM micrograph of single stainless steel wire

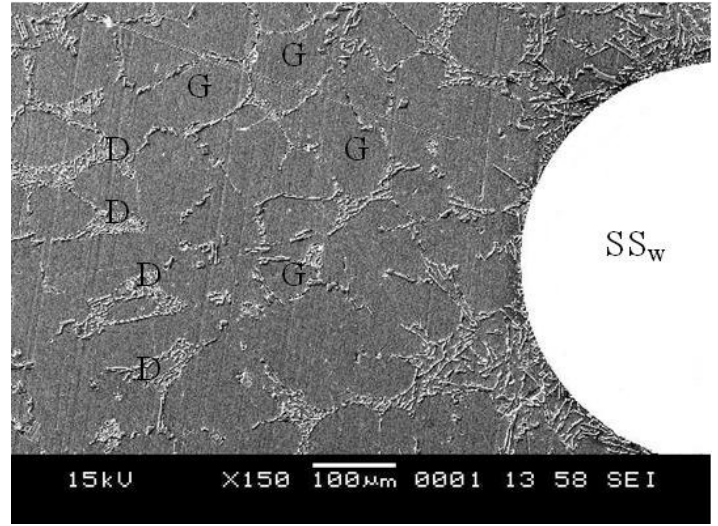

Fig. 3b: Magnified SEM micrograph indicates the morphological evolution around single stainless steel wire 


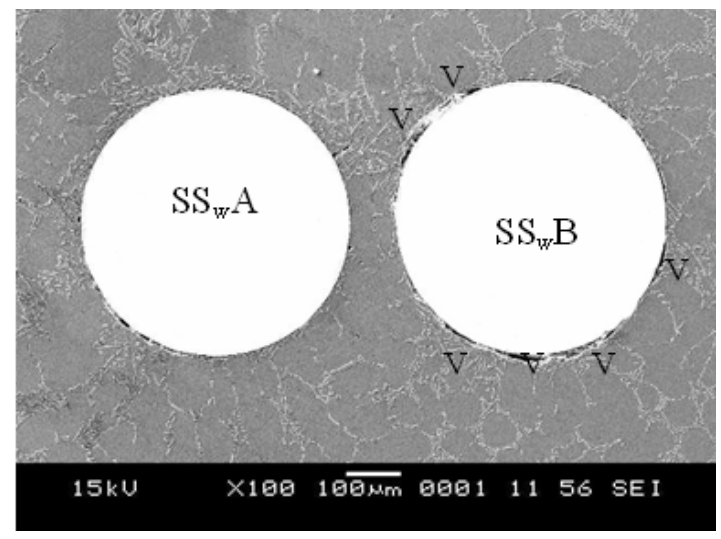

Fig. 4a: SEM micrograph of double stainless steel wires

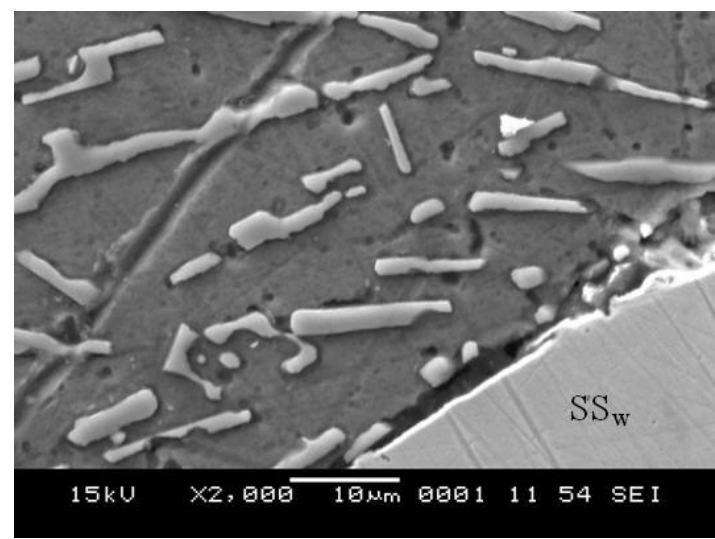

Fig. 4b: Magnified SEM micrograph indicates the coarse acicular eutectic $\mathrm{Si}$ needle shaped particle near to the double stainless steel wires

has undergone evolution to the fine interdendritic eutectic Si particles (denoted as D) and globular structures (denoted as G). Very minimal of both typical porosity and oxide inclusion contents can be observed throughout the Al-Si-Mg matrix alloy.

Figure $4 \mathrm{a}$ shows a micrograph of good wetting $\left(\mathrm{SS}_{\mathrm{w}} \mathrm{A}\right)$ and incomplete wetting $\left(\mathrm{SS}_{\mathrm{w}} \mathrm{B}\right)$ at the double stainless steel wires. Fewer needles shaped eutectic Si particles at the interface and a few voids (denoted by V) can be observed around the interfaces of double stainless steel wires. This condition may be affected by incomplete solidification at the interface owing to the constrained interwire region and lack of vibration.

At higher magnification near to the double stainless steel wires in Fig. 4b, the coarse acicular eutectic Si needle shaped particles are found to be dispersed among the fully developed primary aluminum

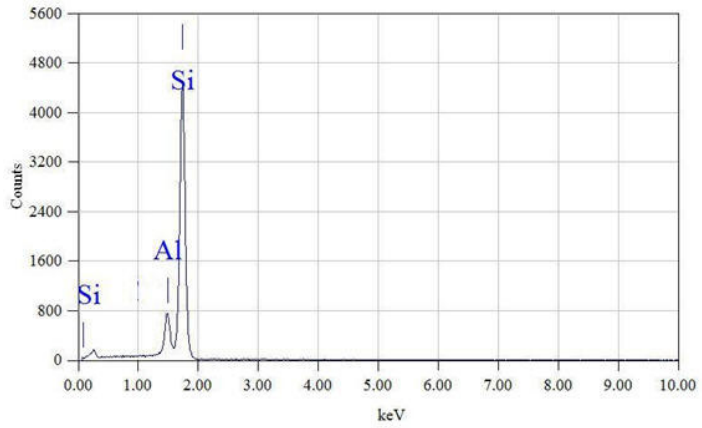

Fig. 5: EDS spectrum of needle shaped eutectic Si particle

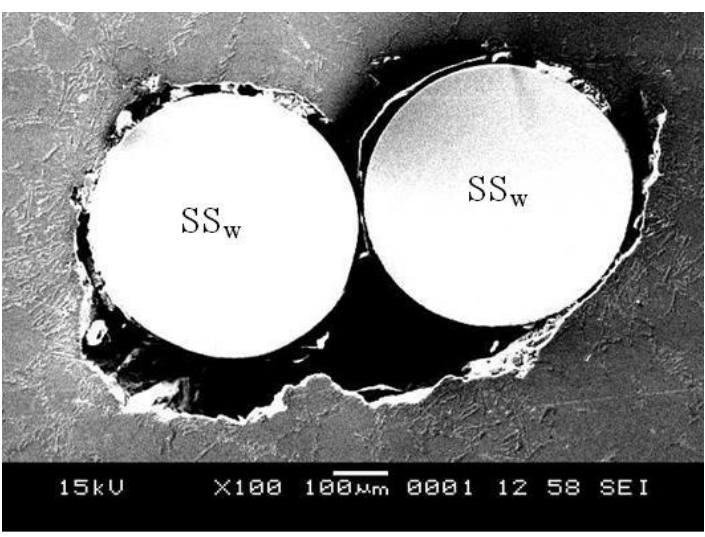

Fig. 6: Very poor wetting associated with large voids around double stainless steel wires

dendrites. This result is in accordance with the previous findings for the without vibration Al-7\% Si alloys ${ }^{[17]}$.

Figure 5 illustrates the EDS spectrum of needle shaped eutectic Si particles which shows that only Al and $\mathrm{Si}$ elements exist and there is no formation of any intermetallic compounds.

Very poor wetting associated with large voids are clearly shown in Fig. 6 due to the intimate contact between the stainless steel wires and thus no contact of $\mathrm{Al}-\mathrm{Si}-\mathrm{Mg}$ matrix alloy in between the stainless steel wires.

Generally, the wetting of metal-metal composites is better compared to metal-ceramic composites. Wetting is favored by the formation of strong chemical bonds at the interface. Good wetting thus often also involves good bond strength at the interface in the final composite. The formation of these bonds may be accompanied by mutual dissolution and/or reaction of the two phases. The latter phenomena are very detrimental to the composite as they bring about a decrease of the mechanical properties ${ }^{[18]}$. 


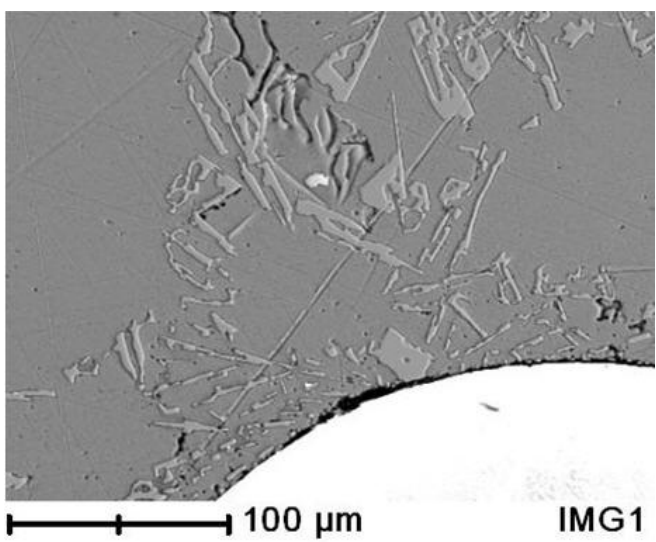

(a)

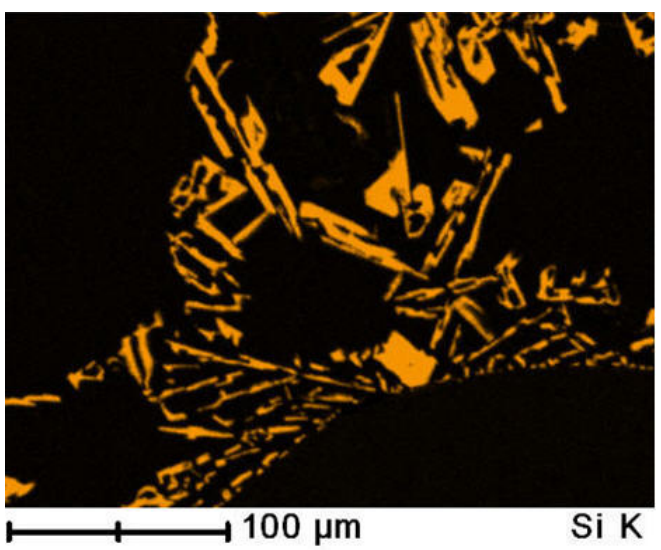

(c)

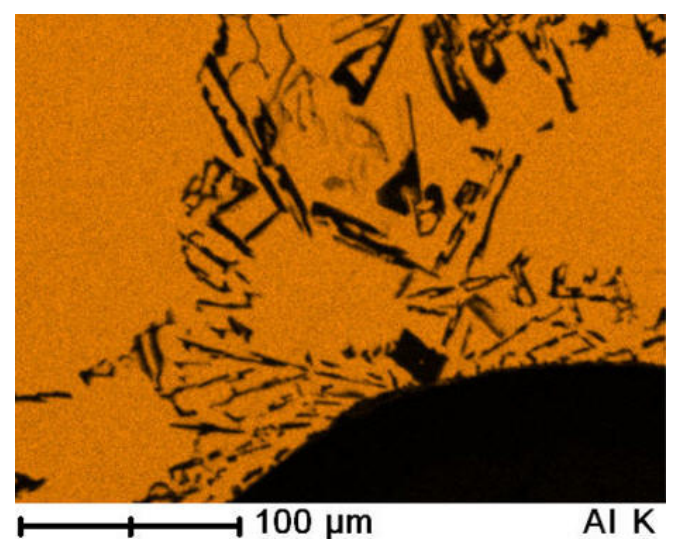

(b)

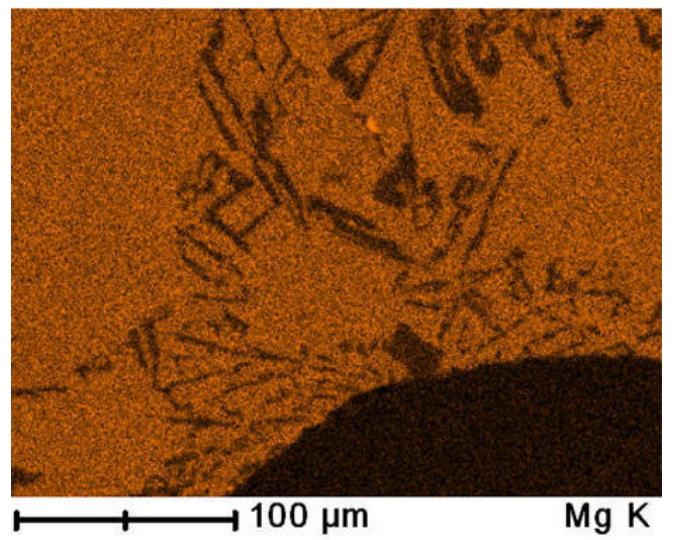

(d)

Fig. 7: (a) SEM micrograph, and EDS mapping of (b) aluminium (Al), (c) silicon ( $\mathrm{Si}$ ), and (d) magnesium (Mg)

\section{CONCLUSIONS}

The following conclusions can be drawn:

- Single stainless wire shows a good wetting condition meanwhile double stainless steel wire shows a good wetting, incomplete wetting and very poor wetting conditions.

- Heavily concentrated of needle shaped eutectic Si structures and a larger size of globular structure can be seen near to and around the single stainless steel wire respectively. These microstructural features may be attributing to the lower solidification rate around the single stainless steel wires compared to the double stainless steel wire.

- Very poor wetting associated with large voids are due to the intimate contact between the stainless steel wires and thus no contact of Al-Si-Mg matrix alloy in between the stainless steel wires.
- Incomplete solidification at the interface of double stainless steel wires is owing to the constrained interwire region and lack of vibration.

- The wettability of solid metals by liquid metals is very important in determining the needed properties of the composite systems. Wetting condition is favoured by the formation of strong chemical bonds at the interface and also by providing appropriate constrained interwire region and vibration during the processing.

\section{ACKNOWLEDGMENT}

The authors are grateful to UniMAP for the financial support under Short Term Grant (Acc. No. 9003-00021).

\section{REFERENCES}

1. Metcalfe, A.G., 1974. Introduction and Review in Composite Materials, Academic, pp: 50-79. 
2. Wu Jiejun, Li Chenggong, Wang Dianbin and Gui Manchang, 2003. Damping and sound absorption properties of particle reinforced Al matrix composite foams. Comp. Sci. Technol., 63: 569-574.

3. Grigoris E. Kiourtsidis, Stefanos M. Skolianos and George A. Litsardakis, 2004. Aging response of aluminum alloy 2024/silicon carbide particles (SiCp) composites. Mater. Sci. Eng., A 382: 351-361.

4. Modi, O.P., Mohini Saxena, B.K. Prasad and A.H. Yegneswaran, 1992. Corrosion behaviour of squeeze-cast aluminum alloy-silicon carbide composites. J. Mater. Sci., 27: 3897-3902.

5. Durrant, G., M. Gallerneault and B. Cantor, 1996. Squeeze cast aluminum reinforced with mild steel inserts. J. Mater. Sci., 31: 589-602.

6. Jae-Chul Lee, Gyeung-Ho Kim, Jung-Ill Lee, HoIn Lee, 1997. Interfacial Reactions in the SqueezeCast (SAFFIL1C)/SAE 329 Al Composite. Metall. Mater. Trans. A, 28A: 1251-1259.

7. Lim, S.C., M. Gupta, L. Ren and J.K.M. Kwok, 1999. The tribological properties of $\mathrm{Al}-\mathrm{Cu} / \mathrm{SiCp}$ metal-matrix composites fabricated using the rheocasting technique. J. Mater. Proc. Technol., 89-90: 591-596.

8. Rohatgi, P.K., J.K. Kim, N. Gupta, Simon Alaraj, A. Daoud, 2006. Compressive characteristics of A356/fly ash cenosphere composites synthesized by pressure infiltration technique. Composites, Part A 37: 430-437.

9. Payan, S., Y. Le Petitcorps, J.-M. Olive and H. Saadaouic, 2001. Experimental procedure to analyse the corrosion mechanisms at the carbon/aluminum interface in composite materials. Composites, Part A 32: 585-589.
10. Rajan, T.P.D., R.M. Pillai and B.C. Pai, 1998. Review: Reinforcement Coatings and Interfaces in Aluminum Metal Matrix Composites. J. Mater. Sci., 33: 3491-3503.

11. Anita Olszówka-Myalska, Janusz Szala and Jan Cwajna, 2006. Characterization of iron aluminides formed in situ in an aluminium matric composite. Mater. Charac., 56: 379-383.

12. Abenojar, J., M.A. Martinez and F. Velasco, 2006. Effect of the boron content in the aluminum/boron composite. J. Alloys Compd., 422: 67-72.

13. Tiwari, S.N., A.N. Tiwari and V. Gopinathan, 1987. High Temperature Interfacial Studies in Aluminum-Stainless Steel Composites. J. Mater. Sci., 22: 2680-2684.

14. Bhagat, R.B., 1989. Growth Kinetics of Interface Intermetallic Compounds in Stainless Steel Fiber Reinforced Aluminum Matrix Composites. J. Mater. Sci., 24: 1496-1502.

15. Mandal, D., B.K. Dutta and S.C. Panigrahi, 2006. Microstructure and mechanical properties of Al$2 \mathrm{Mg}$ alloy base short steel fiber reinforced composites prepared by vortex method. J. Mater. Sci., 41: 4764-4770.

16. Hashim, J., L. Looney and M.S.J. Hashmi, 1999. Metal Matrix Composites: Production by the Stir Casting Method. J. Mater. Proc. Technol., 92-93: $1-7$.

17. Jian, X., T.T. Meek and Q. Han, 2006. Refinement of Eutectic Silicon Phase of Aluminum Al-7\%Si Alloy using High-Intensity Ultrasonic Vibration. Scripta Mater., 54: 893-896.

18. Delannay, F., L. Froyen and A. Deruyttere, 1987. Review; The Wetting of Solids by Molten Metals and Its Relation to the Preparation of Metal-Matrix Composites. J. Mater. Sci., 22: 1-16. 